\section{Neuroscience Education}

\section{P.069}

Pediatric neurology subspecialty education development in a resource limited setting

A Mineyko (Calgary)* L Day (Calgary) E Kumbakumba (Mbarara) D Santorino (Mbarara) D Boctor (Calgary)

doi: $10.1017 / \operatorname{cjn} .2019 .169$

Background: In 2013, the University of Calgary (UofC) - Mbarara University of Science and Technology (MUST) Pediatric Education Program was established when the Pediatric Department in Mbarara, Uganda identified a need for enhanced education in pediatric subspecialty areas. We report on the experience of developing the pediatric neurology subspecialty curriculum. Methods: Pre-visit meetings established mutually agreed upon objectives and learning activities that were implemented over 2-week periods in 2015 and 2018. Pre and post-tests were administered to MUST Pediatric residents. Mean differences in test scores were compared using a Student t-test. Residents provided written feedback following the end of the second visit. Results: A pediatric neurologist (AM) visited MUST (2015 and 2018) to deliver the curriculum. The second visit was accompanied by a senior UofC Pediatrics resident (LD). Eight and 14 residents at MUST participated in the curriculum in 2015 and 2018, respectively. Neurology test scores improved in 2015 from a mean of $43 \%$ to $61 \%(p=0.011)$ and in 2018 from $53 \%$ to $84 \%(p<0.00001)$. Teaching sessions were well received by MUST residents. Conclusions: Collaboration between UofC faculty and MUST established an effective pediatric neurology curriculum that was well-received by residents.

\section{Neurovascular, Stroke and NeUROINTERVENTIONAL}

\section{P.070}

Thrombolysis without large vessel occlusion in a child with acute arterial ischemic stroke

IE Hanes (Ottawa)* SL Orr (Calgary) J Davila (Ottawa) A Kirton (Calgary) E Sell (Ottawa)

doi: $10.1017 / \operatorname{cjn} .2019 .170$

Background: Stroke is a rare neurological disease in children, with an annual incidence of 2 - 13/100,000 children per year. Pediatric stroke is associated with significant morbidity and mortality lasting many decades. Diagnosis of pediatric stroke is challenging and often delayed, limiting options for acute intervention, and the pharmacological and mechanical recanalization strategies that have revolutionized adult stroke remain undefined in children. Clinicians are left to draw conclusions from other retrospective cohort studies or case reports and extrapolate adult guidelines to the pediatric population. The TIPS trial eligibility criteria are often used in clinical practice, despite not being validated for this purpose. We present here the case of a healthy 14 year old male who was treated with intravenous tissue plasminogen activator (IV tPA) for a presumed arterial ischemic stroke without large vessel occlusion on neuroimaging. Methods: Retrospective chart review Results: Not applicable Conclusions: Following the administration of IV tPA, the patient made a full recovery. While we do not recommend the routine use of IV tPA for treatment of presumed large vessel or small vessel in children, we suggest that the decision to proceed with IV tPA be considered on a case-by-case basis.

\section{Other Child Neurology}

\section{P.071}

Clinical and demographic predictors of stress in parents of children with genetically determined leukoencephalopathies

EA Dermer (Montreal)* A Spahr (Montreal)* L Tran (Montreal) A Mirchi (Montreal) F Pelletier (Quebec City) K Guerrero (Montreal) The Leukodystrophy Family Impact Research Group () G Bernard (Montreal)

doi: 10.1017/cjn.2019.171

Background: Genetically-determined leukoencephalopathies comprise a rare group of inherited white matter disorders. The vast majority are associated with a progressive disease course and early death. This study seeks to determine the clinical and demographic correlates of stress in parents of leukodystrophy patients, for future clinical guidance. Methods: A cross-sectional study including 36 families was performed. Children aged 1 month to 12 years with a diagnosed leukodystrophy or genetically-determined leukoencephalopathy were included. 31 mothers and 24 fathers completed the Parental Stress Index, 4th edition (PSI-4). One demographic questionnaire was completed per family. Clinical data was gathered within 6 months of the questionnaires. Statistical analysis was performed with total stress (TS) scores as the primary outcome. Results: Mothers and fathers had comparable TS scores. No clinical or demographic factors predicted the father's TS score. Greater ambulatory impairment, using the GMFCS scale, correlated to lower TS scores in the mother. Conclusions: The progressive nature of these conditions makes it such that anticipating a child's inability to walk may cause more stress for mothers than a child's actual inability to ambulate. The inability of all other variables to predict total stress highlights a need for individualized approaches when addressing stress in these families.

\section{P.072}

Fetal alcohol spectrum disorder - is this a ciliopathy?

JL Urquhart (Edmonton)* HR Goez (Edmonton)

doi: 10.1017/cjn.2019.172

Background: Fetal alcohol spectrum disorder (FASD) is used to describe the spectrum of birth defects due to prenatal alcohol exposure; these include craniofacial abnormalities and intellectual disabilities. The prevalence of FASD is estimated at 1 in 100. Diagnostic criteria include distinct facial features, neurodevelopmental deficits and confirmation of alcohol use during pregnancy. Unfortunately, often criteria are missed or absent. No biochemical marker is available for screening and diagnosis of FASD that is easy, accurate and cost-effective. 